\title{
Italique
}

Poésie italienne de la Renaissance

XX $\mid 2017$

Varia

\section{Gli alberi e il «libro». Percorsi dell’ Arcadia di Sannazaro}

\section{Massimo Danzi}

\section{(2) OpenEdition}

\section{Edizione digitale}

URL: http://journals.openedition.org/italique/458

DOI: $10.4000 /$ italique.458

ISSN: 1663-4438

\section{Editore}

Librairie Droz

\section{Edizione cartacea}

Data di pubblicazione: 1 ottobre 2017

Paginazione: 119-148

ISBN: 978-2-600-05818-6

ISSN: 1423-3983

Notizia bibliografica digitale

Massimo Danzi, « Gli alberi e il «libro». Percorsi dell' Arcadia di Sannazaro », Italique [Online], XX | 2017, online dal 01 octobre 2019, consultato il 22 janvier 2020. URL : http://journals.openedition.org/ italique/458 ; DOI : 10.4000/italique.458

(c) Tous droits réservés 


$$
\text { Mas S I M D A N Z }
$$

GL I A L B E R I E I L «L I B R O».

PERCORSI DELL' $A R C A D I A$

D I S A N N A A R O 



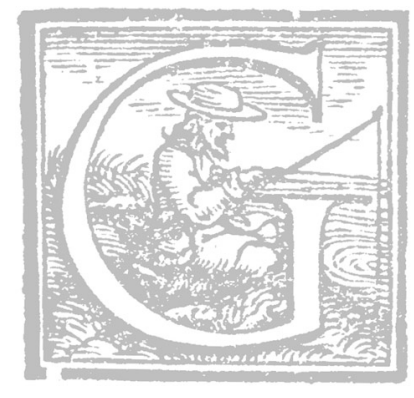

li studi sull'Arcadia di Sannazaro degli ultimi sessant'anni evidenziano alcune linee di ricerca, fissate le quali, vorrei, in queste pagine, fornire un'interpretazione di alcuni elementi del prosimetro, che giudico paradigmatici della scrittura sannazariana. L'arco della bibliografia considerata (1952-2013) $)^{\mathrm{I}}$ è sufficientemente ampio per essere, pur con inevitabili approssimazioni, rappresentativo e, nella prima parte di questo contributo, sarà oggetto di un breve percorso teso a evidenziare le principali angolature da cui il testo è stato affrontato nello sforzo di chiarirne la lettera, le allusioni politico-letterarie e a volte i suoi "enigmi".

In queste pagine ragiono come se l'Arcadia sia leggibile nella sola redazione definitiva, la «summontina» del I504. ${ }^{2}$ Non ricorrerò cioè, in genere, a stati del testo precedenti e ricorrerò poco anche alla storia del genere o allillustrazione storica degli eventi cui il testo fa riferimento, piani privilegiati da molti importanti studi che contribuiscono a un'interpretazione scientifica del testo senza tuttavia, come è ovvio, poterne esaurire la ricchezza. Sul piano esegetico, lo 'svelamento' di una fonte di Sannazaro, l'identificazione storica di fatti o personaggi o, per esempio, il rinvio per un nome di pastore alla tradizione dell'onomastica bucolica sono in sé elementi importanti dell'operare del poeta, e tuttavia non dicono ancora niente del dialogo che l'autore intesse con la tradizione e delle scelte che compie; non chiariscono - mi pare - l'uso letterario di quegli istituti o, nella fattispecie, il 'sistema' onomastico che regge il libro, per ricordare una delle vie, che a me pare più promettente nell'interpretazione dell'Arcadia. ${ }^{3}$ Lettore negli anni io stesso di molti commenti, confesso di essere sempre piu imbarazzato di fronte a 'nudi' rinvii testuali che non si accompagnano a pur sobrie valutazioni critiche dei materiali convocati dai confronti. L'interpretazione comincia infatti dopo tali anamnesi (pur - ripeto - fondamentali), come sempre più ci ricorda anche il pubblico dei nostri allievi alle prese con le difficoltà di 'capitalizzare' miriadi di puntuali osservazioni in funzione di una interpretazione che non sia impressionistica né meramente classificatoria.

In queste pagine considero prima quanto gli studi sull'Arcadia ci hanno offerto e, dialogando con alcuni di essi, avanzo poi una proposta di «lettura» del testo. 
I. Tre direzioni mi paiono prevalere negli studi sull'Arcadia degli ultimi sessant'anni. La prima è d'ordine linguistico-filologico e, senza soluzione di continuità con i principali interventi tra Otto e Novecento (diciamo Torraca, Scherillo per l'edizione del testo; Percopo e Carrara per l'ambiente storico-letterario e il 'genere'), risale, nel suo segmento 'moderno', ai contributi linguistico-filologici di Gianfranco Folena, da una parte (1952), e di Maria Corti, dall'altra (1956 e sgg.). Nel I96I l'edizione critica del testo (o meglio della sua ultima 'redazione') di Alfredo Mauro offriva quella che è ancora oggi l'unica edizione scientifica a disposizione, a questo non potendo pretendere la pur meritoria, e ricchissima per il commento, edizione dello Scherillo (I888). Questa linea - è noto - prosegue, con importanti novità e revisioni, fino ai numerosi e fondamentali studi filologici di Gianni Villani (1989, I99I e 2009) o alle prospezioni sulla «summontina» del I504 della Charis Marconi (1997), che hanno posto le basi per una nuova edizione. ${ }^{4}$

Una seconda direzione di studì è consistita nel chiarire un testo spesso enigmatico quando non decisamente criptico. Lasciando da parte $i$ commenti, che evidentemente si prefiggono questo scopo, il segmento 'moderno' di questi studi risale alla monografia di Marco Santagata (1979) e agli studi che, dialogando spesso con lui, hanno dato soprattutto Marina Riccucci (200I), Enrico Fenzi (2008 e 2009) e Isabella Becherucci (2014). Se la Becherucci si è mossa su altro piano (che vedremo), Riccucci e Fenzi hanno interpretato il testo sullo sfondo della stagione aragonese, ricostruita con novità di voci poetiche da Santagata per quanto atteneva alla congiuntura storico-politica: la Riccucci esplorando, attraverso la stratigrafia del testo, particolarmente l'isotopia legata al tema dei «lupi» e del «ladro», ${ }^{6}$ Fenzi sottolineando la dimensione politica del quadro tracciato da Sannazaro in cui coglie una denuncia contro lo strapotere dei funzionari catalani chiamati a Napoli dagli Aragonesi. Lo stesso autore dell'Arcadia sarebbe, a gindizio di Fenzi, «assai meno filo-aragonese» di quanto finora ritenuto. ${ }^{7}$

Tra questi studiosi, Santagata è forse quello che mantiene un maggior equilibrio tra 'valori' letterari dell'Arcadia e quelli che per comodità diremo 'storico-politici. ${ }^{8}$ Dalla sua monografia in poi, lo sfondo (già denunciato dalla scuola storica) del contesto napoletano, con lo scontro in atto tra dinastia e grandi feudatari, sembra aver imposto sempre più le sue ragioni nell'interpretazione del testo, fino alle parole esplicite di Fenzi che, richiamando la vessatoria politica fiscale aragonese nei 
confronti dei piccoli e grandi feudatari del regno (di cui fanno le spese gli stessi Sannazaro con le cave di Agnano o $i$ De Jennaro per il feudo alle Fratte), afferma chiaro:

Confesso in limine che nei confronti delle tradizionali alternative critiche mi sento naturalmente inclinato ad approfondire quella che privilegia la dimensione etico-politica dell'Arcadia e dunque cerca di leggerla sullo sfondo degli ultimi due drammatici decenni della Napoli aragonese. Non ho dubbi, sul fatto che Sanazaro interiorizzi proprio quel dramma, di cui era diretto e partecipe testimone, e che ne faccia il vettore fondamentale della sua simbolica vicenda. ${ }^{9}$

Questa linea di ricerca ha avuto il merito di proporre un'Arcadia assai meno rousseauiana e idillica di ciò che poteva parere, toccando e correggendo singoli aspetti della cronologia (ultima la Riccucci, che ipotizza la composizione del congedo A la sampogna a ridosso della stampa del Is04) o dell'interpretazione di brani che, per essere politicamente 'esposti', erano apparsi enigmatici fin dalle egloghe di più antica composizione (I, II, VI e X). E noto tuttavia che l'oscurità del testo, cui deve in qualche luogo rassegnarsi anche l'ultimo commentatore, è un dato 'fisiologico' non spiegabile solo con la prudenza che il contesto politico poteva richiedere ma connesso direttamente a un genere che autori $e$ commentatori si accordano a ritenere, fin dal Medioevo, difficilmente comprensibile senza una chiave interpretativa. Cosi la pensa il Petrarca, che all'inizio delle Sine nomine ricorda come il suo Bucolicum carmen appartenga a un «poematis genus ambigui [...] paucis intellectum» e cosi anche, qualche anno dopo, Benvenuto da Imola che, a proposito delle eclogae virgiliane, ritiene anche più esplicitamente «impossibile quod aliquis intelligat bucolica nisi babeat aliquid ab illo qui composuits. ${ }^{\text {Io }} \mathrm{Di}$ ciò lo stesso Sannazaro si mostra cosciente nell'Arcadia, direttamente o per bocca dei suoi pastori. ${ }^{\text {I }}$

Non importa entrare qui più a fondo nelle novità e divergenze recate dal dialogo di Fenzi, e soprattutto della Riccucci, con Santagata e altri; ma certo la proposta dello studioso genovese di identificare la «famelica genia di genti strane, inique, inexorabili») denunciata da Caracciolo nell'ecloga XI con quella classe di impiegati e cortigiani catalani, che a Napoli $i$ monarchi avevano fatto venire e che aveva finito per avere importanti ruoli economici e amministrativi a corte - e dunque l'accento posto sui monarchi piuttosto che sui feudatari e baroni del Regno 
(com'era tradizione) - mostra come ancora l'interpretazione storica del testo possa subire nuove accelerazioni. ${ }^{{ }^{2}}$ Una interpretazione che Fenzi vede confermata dal «sostanziale silenzio che l'Arcadia riserva ai re aragonesi» e che incrina l'immagine del «fedele gentiluomo che segue il proprio re nell'esilio e gli resta vicino sino alla morte» proponendoci un Sannazaro assai meno "aragonese» di quanto finora creduto se non addirittura abitato da una "vena filo-baronale e anti-monarchica». ${ }^{\mathrm{I}}$ Le direzioni ricordate, quella linguistico-filologica e questa più storicopolitica, non corrono ovviamente separate. Nella drammatica congiuntura storica napoletana degli anni'80-'90, la genesi e crescita del testo, durata più di un decennio, acquista sfumature diverse a seconda del rapporto che riflette con la realtà. Ciò riguarda naturalmente una serie di temi, come, per esempio, quello del rapporto tra Napoli e l'Arcadia (per Santagata di «alternativa» o per Fenzi «nulla dipiu del 'doppio' altrettanto angoscioso dell'unico mondo possibile») ${ }^{14}$ o l'altro del corollario rappresentato dall'«esilio» di Sincero, ${ }^{15}$ risolto poi solo nella «seconda» redazione con il «ritorno» a Napoli narrato nei due ultimi capitoli dell'Arcadia.

Con accenti diversi, queste due direzioni di studi si sono imposte, mi pare, su ogni altra 'lettura', segnando in profondità gli studi sull'Arcadia. Senza arretrare al commento del Carrara, le ritroviamo capitalizzate nei più recenti commenti di Espamer (1990), Marino (2004) e Vecce (2013), dove a volte sono accompagnate da una valutazione critica dei materiali convocati. ${ }^{16}$

2. C'è però anche una terz̧a e più eterogenea direzione di studî, che va ricordata prima di passare alla pars costruens di questo intervento. E fatta di contributi storico-letterari più generali e ricapitolativi (dall'innovativo capitolo della "Garzanti» firmato da De Robertis nel I966 ai saggi di Villani: da quello per la Letteratura italiana Einaudi, 1992 fino all'ultimo - per ora - del 2009), di letture più metrico-formali (a partire dal panorama di De Robertis sull'ecloga volgare fino al Quattrocento: 198I) e a seguire con studi dedicati a singoli aspetti: dall' «artificiosità» e dalle "figure» del genere indagate da Parenti (Parenti 1987) alla fortuna del prosimetro a Napoli (Vecce 2000), alla morfologia del «libro» pastorale (Carrai 2006) fino a quelle di singoli temi e topoi (Muñiz, Muñiz, Rinaldi, Villani 2009, ecc.). Se fuori da compilazioni storico-letterarie, una dimensione «globale» attenta alla struttura del testo e alle modalità della narrazione non sembra facile a trovarsi (bisogna rinviare ai saggi di Tateo, Saccone, 
Aricò o Haywood, Rinaldi o all'interpretazione psicanalitica e attualizzante di Gajetti), abbiamo invece 'assaggi' su singoli aspetti. David Quint ha inserito l'opera in una dimensione 'orfica' e cosi, poi, particolarmente la Caracciolo Arico; Prandi ha sottolineato l'importanza dell'ecfrasis; Tateo e Muñiz Muñiz hanno toccato aspetti dell'onomastica e del tema della descriptio puellae. Singole 'finestre' sulla fortuna europea dell'Arcadia (soprattutto francese e spagnola) hanno poi fornito Boudard 1980, Vecce 1999, Muñiz Muñiz 2007 o Gargano 2009. In tal modo, nel 2012, Isabella Beccherucci ha tirato le fila approfondendo alcune linee di lettura presenti negli studi: penso in particolare al rapporto prosa-poesia, indicato da De Robertis come fondativo, con il corollario che reca della continuità tra «prima» e «seconda» parte del prosimetro e, sul piano dei personaggi, del particolare rapporto di specchiamento tra Carino e Sincero, controfigure dei due massimi poeti volgari a Napoli: il Cariteo e il Sannazaro. ${ }^{17}$

Per profondità e scandaglio della dimensione letteraria e «allusiva» del testo, nonché per la data che reca (1983), svetta a mio parere sull'Arcadia soprattutto il contributo di Giuseppe Velli. Dopo Saccone e Tateo, Velli centra il tema fondamentale della «struttura» dell'Arcadia dando complessità di rappresentazione ai «processi attraverso i quali l'opera si costituisce» $(\not . I X)$ e mettendo a fuoco, o correggendo, precedenti interpretazioni attraverso una 'lettura' attenta come mai prima alla dimensione allusiva, costituita da rinvii interni e esterni all'opera di Sannazaro. Velli non mira a un'interpretazione complessiva del prosimetro, ma vi si avvicina scegliendo un piano, quello costituito dalla 'letterarietä' del testo, che fa leva sulla strumentazione topica del genere, che pare anche a me di rilievo.

Gli interventi che ho raccolto in queste tre classi, con una esemplificazione che spero decentemente rappresentativa, hanno contribuito non poco a maturare una consapevolezza nuova della testualità di Sannazaro, che $i$ commenti hanno fatto avanzare sul piano della lettura puntuale misurando l'Arcadia sulla base delle esperienze poetiche contemporanee: da quella latina del Pontano alle volgari di Cariteo, De Jennaro o Galeota fino ai meno noti Caracciolo o De Petruciis. Ma se la situazione è questa, bisogna riconoscere che su altri fronti manchiamo invece ancora fortemente. Non abbiamo, pur dopo gli studi del Folena e della Corti, un studio complessivo sullo «stile» della «summontina», né del «linguaggio» pastorale sul lungo periodo. Passato relativamente sotto 
silenzio, con le eccezioni di Tateo, di Brugnoli e della Muñiz. Muñiz, mi pare il piano (capitale nella bucolica) dell'onomastica e relativamente disertato quello di una interpretazione 'critica' del testo. In questo ultimo ambito, un poco si perpetua ancora, a mio modo di vedere, il «curioso destino» che Maria Corti dipingeva per l'Arcadia, ormai mezzo secolo fa, invitando a «far parlare l'opera»:

riconoscimento immediato dei contemporanei, notevolissimo influsso sul genere arcadico nella letteratura italiana, in quelle spagnole, portoghese, inglese, francese; eppure chi cerchi entro il grande mare della saggistica contemporanea un'indagine di natura squisitamente critica dedicata al capolavoro del Sannazaro, trova ben poco al di là di qualche celebrazione rituale d'ufficio nel centenario dello scrittore. ${ }^{18}$

In mezzo secolo le cose anche sull'Arcadia, sono naturalmente cambiate e se il fronte di una riflessione che oltrepassi e traduca in termini 'critici' le acquisizioni storiche e filologiche non è andato forse di pari passo con queste, pure abbiamo assistito a una pluralità di accessi al testo, che hanno interessato la struttura dell'opera, $i$ suoi diversi stati redazionali, l'elaborazione e le direzioni linguistiche imboccate da Sannazaro, l'assetto metrico, la polifonia delle 'voci' o, infine, le questioni afferenti al 'genere' (di arrivo): il prosimetro. Più dimenticato, o per lo meno rimasto inerte all'interpretazione, mi pare il piano invece configurato da topoi, motivi e strutture di questo linguaggio, ${ }^{19}$ quell'outillage insomma che rende il poeta bucolico immediatamente riconoscibile e fonda lo specifico di una scrittura fortemente e sostanzialmente 'allusiva' com'è quella dell'Arcadia.

Credo anch'io con Yves Bonnefoy, prefatore del bel commento di Gerardo Marino edito per le «Les Belles Lettres» (2004), che l'Arcadia offra molto su questo versante ("L'Arcadia me paraît, comme à quelques autres d'ailleurs, moins un poème qu'une réflexion sur la poésie») $)^{20}$ e certo l'osservazione del poeta francese è funzione diretta del taglio che Marino ha dato al suo commento, mettendo al centro della lettura, in particolare nell'Introduzione, la dimensione 'allusiva' generata dai rinvii alle fonti sannazariane. L'importanza di questo piano è stata sottolineata più recentemente da un bell'intervento di Rinaldi (sul quale ritornerò, perché è il precedente più vicino all'ipotesi che presento in queste pagine) e da uno successivo di Villani, nel contributo suo più aperto all'esegesi del prosimetro, il quale afferma: 
Sembra insomma che si osservino due linee di fuga nell'Arcadia, separate ma interdipendenti: una, meglio misurabile, dai luoghi di una immaginazione offuscata in una stagione al tramonto, l'altra metatestuale, concernente il rapporto dell'autore col suo libro. ${ }^{2 \mathrm{I}}$

Vedremo che su questa seconda via, aperta dai saggi sannazariani di Tateo e di Velli, si può andare ancora più avanti.

3. Vorrei allora partire dalla costatazione di Bonnefoy che mi pare tra quelle che più danno ragione della natura eminentemente letteraria del testo, per entrare in uno «spazio» di riflessione ancora relativamente vergine, attraverso tre temi osservati dalla specula della edizione del I 504. Il primo tema concerne la presenza nell'Arcadia del noto mito di Orfeo, noto appunto ma non sufficientemente messo in relazione con la «costruzione» del libro; il secondo è il topos, altrettanto noto, della 'scrittura sulla corteccia', diffusissimo in ambito pastorale e tuttavia rimasto altrettanto inerte nell'interpretazione della «testualità» sannazariana; il terzo, strettamente connesso ai due che precedono, valorizza la dimensione metatestuale e metapoetica dell'Arcadia, un piano forse subliminale ma che direttamente partecipa all'originalità del libro perché conferma la coscienza che l'autore ebbe del suo "fare», al di là dei luoghi cui normalmente si rinvia e, in particolare, al di là del congedo A la sampogna. ${ }^{22}$

\section{Orfeo}

Partiamo dalla presenza di Orfeo nell'Arcadia, sul quale si sono fermati Quint dialogando con Saccone, e poi, in vari interventi, la Caracciolo Arico.. ${ }^{23}$ Per esso vale quanto, introducendo l'Orfeo di Poliziano, osservava Antonia Tissoni Benvenuti invitando a separare il motivo frequente in tutti $i$ tempi dell' «Orfeo poeta cantore» da quello di Orfeo e Euridice, che è "altra cosa». ${ }^{24}$ Sannazaro utilizza $i$ due motivi facendo di Orfeo un modello, forse il principale, della «poetica» perseguita con l'Arcadia. Questo, che mi appare un tratto peculiare dell'opera, riceve - credo - nuovo vigore dallo spazio che al tema conferisce Poliziano, ricomponendo $i$ due aspetti nel catalogo degli esempi mitologici dei Nutricia, poemetto che nelle Sylvae proprio celebra il ruolo civilizzatore della poesia ( $v v$. I27-3I e 285-3I7). ${ }^{25}$ Seppure il tema è in tutto ben polizianesco, e anzi alla metà degli anni '70 
la Fabula di Orfeo è responsabile del trasferimento del mito in ambito pastorale, sono $i$ Nutricia che, composti nell'ultimo quarto di secolo ma editi solo nel I49I, rinfrescano e forniscono, e non soltanto a Sannazaro, una più sistematica ragione del tema orfico. Mentre nella tradizione pastorale Orfeo è principalmente il cantore che trascina la natura, nell'Arcadia questo aspetto si coninga col tema euridiceo della discesa agli inferi, dove la catabasi, se pur fallisce il ricupero della donna, sembra avere all'inizio anche una funzione consolatoria. Andrà però studiata la disseminazione del tema nell'Arcadia, cioè l'apporto che reca alla «costruzione» del libro.

Non è evidente, ma il tema appare fin dall'inizio dell'Arcadia. Nel catalogo di piante che Sannazaro presenta sul monte Partenio, e per il quale si sono indicate senza troppo distinguere le fonti fin dallo Scherillo, ${ }^{26}$ svetta tra tutte il «cipresso» (I I-s):

Ma fra tutti, nel mezzo, presso un chiaro fonte, sorge verso il cielo un dritto cipresso [...] nel quale nonché Ciparisso, ma, se dir conviensi, esso Apollo non si sdegnarebbe essere transfigurato.

L'indicazione isolata, del cipresso rinvia, tra tutte le fonti, al solo Ovidio, Metamorfosi x 86-106, ove l'albero chiude, come in Sannazaro, l'elenco delle piante presenti sul Partenio, per un verso sigillando il racconto che precede di Orfeo ( $v v . I-85)$ e per un altro inescando, come in Sannazaro, il mito di Ciparisso ("Adfuit buic turbae metas imitata cupressus, / nunc arbons, ecc., I06). Ad Orfeo, dunque, l'Arcadia allusivamente rinvia da subito e l'allusione va poi prendendo concretezza nel seguito dell'opera. Nell'ecloga IV, in forma di sestina lirica, uno dei pastori, rassicurato dall'altro, s'identifica con Orfeo. Il motivo compare annunciato da Logisto (I6-I8):

piangendo in rime,

sempre in fiamme son visso, e col mio pianto

ho pur mosso a pietà gli alberi e i sassi

e si fa esplicito con Elpino, che per la prima volta vorrebbe emulare il modello. ${ }^{27}$ Presente anche in altri poeti aragonesi, ${ }^{28}$ il tema non ha però mai il carattere sistematico dell'Arcadia, che lo declina narrativamente facendone una tessera della costruzione 'romanzesca'. Ricompare poi esplicitamente nell' ecloga XI, inescato da Ergasto che piange la morte 
della madre Massilia (dopo che la v aveva pianto quella del padre Androgeo). L'analogia ci dice, insomma, che Ergasto perde Massilia similmente ad Orfeo, che aveva perso Euridice. È preludio al finale funebreelegiaco, replicato dal pianto di Barcinio per la morte di Filli, moglie di Meliseo (egl. XII). L'ultima apparizione esplicita del tema è tuttavia affidata all'ecl. XI, monodica e non dialogica com'era la XII. Nell'ecloga, intercalare e parzialmente modulata sulla trenodia di Mosco per Bione (Id. III), Ergasto ribadisce, citandone due volte il nome, l'ambizione che era già stata di Elpino ad identificarsi con Orfeo. ${ }^{29}$

Le due ultime parti dell'Arcadia (XI-XII) ci consegnano il ritorno di Sincero a Napoli: prima semplicemente immaginato sull'onda del canto di Fronimo e Selvaggio (ecl. x), poi attuato con la guida di due ninfe, attraverso un percorso sotterraneo che prelude all'incontro con Cariteo e Summonte, poeti ai quali è affidata l'ecloga conclusiva. Come aveva già visto Tateo, l'incontro induce certo a considerare l'Arcadia anche come un viaggio verso la poesia, ${ }^{30}$ di cui Selvaggio aveva messo in discussione l'esistenza (ecl. I 25-27):

A dire il vero, oggi è tanta l'inopïa

di pastor che cantando all'ombra seggiano che par che stiamo in Scizia o in Ethiopia

e che ora - siamo alla chiusa della prima redazione - è invece costretto a riconoscere (ecl. $\left.\times I_{-3}\right)$ :

Non son, Fronimo mio, del tutto mutole, com'uom crede, le selve, anzi risonano, tal che quasi all'antiche ugual riputole.

I due brani, che aprono e chindono la prima redazione, possono esser visti come una altercatio, un canto amebeo nel canto amebeo, cui l'autore pone fine col congedo A la sampogna, esattamente come un giudice fa nei contrasti fra pastori. Ed $\grave{e}$ in questo finale che il mito di Orfeo riaffiora, questa volta strutturalmente, prima nella variante virgiliana della catabasi poi in figura di ecfrasis con le ninfe ritratte a tessere su un panno la vicenda di Euridice (pr. XII I7). Ma Sannazaro è maestro di allusività e non gli si farà torto a leggere un ultimo rigurgito del tema anche nel topos virgiliano del «durus arator», che appare proprio nel momento in cui il poeta intende deporre per sempre la zampogna. Il brano del congedo A la sampogna, 3: 
a me conviene da le mie labbra disgiungerti [...] come il duro aratore il quale dagli alti alberi inanzi tempo con tutti i nidi si affretta a prendere $\mathrm{i}$ non pennuti ucelli

è stato da tempo ricondotto a Virgilio, ma di nuovo senza - mi pareavvertirne la funzione strutturale che ha nelle Georgiche, dove chiude - vera sfraghís dell'episodio - il racconto di Orfeo. ${ }^{3 \mathrm{I}}$ Annunciato cosi fin dall'esordio, Orfeo segna in vario modo anche la chiusa dell'opera, $e$ non soltanto nell'ultima definitiva redazione. Per la discrezione che lo caratterizza e la simmetria che istituisce con l'esordio, il congedo è quasi una 'dissolvenza', dopo che il tema è giunto ormai - possiamo dire - a saturazione. Sarà troppo, allora, vedere in questo finale, segnato s'è visto da memorie diverse, ${ }^{32}$ un'influenza esercitata, oltre che dall'alta esemplarità dei Nutricia di Poliziano, anche dall'esercizio pastorale di Boiardo, la cui interferenza con Sannazaro è stata ragionevolmente ipotizzata proprio al tempo delle ecloghe latine, sullo sfondo della guerra tra Ferrara e Venezia? Con soluzione che a me pare unica entro la tradizione latina e italiana del genere, Boiardo chiude infatti entrambe le raccolte bucoliche con testi intitolati a Orfeo, l'uno - nei Pastoralia - a cantare Ercole d'Este, l'altro - nelle Pastorale - in lode prima di Alfonso il Magnanimo poi del nipote Alfonso di Calabria, e a entrambi $i$ testi affida - esattamente come fa Sannazaro - anche la conclusione della propria esperienza pastorale annunciando la transizione ad altro registro poetico più elevato. ${ }^{33}$

\section{Metatestualità}

Non mi pare che gli studî abbiano dato la giusta attenzione alle sparse osservazioni metatestuali che si incontrano nel prosimetro. Il tema accennato, ma non sviluppato, da Bonnefoy ricompare nel saggio che Marino ha dedicato agli itinéraires dell'Arcadia nel 2007. Ma anche qui si può credo andare avanti. Vediamo un episodio singolarmente passato sotto silenzio fin dal commento dello Scherillo e poi nei successivi e moderni: la storia della poesia pastorale che appare nella prosa X.

Il brano chiudeva la prima redazione innalzando di fatto il tono del prosimetro, attraverso due elementi, che Sannazaro ba cura di sottolineare nel par. 20: I) la rivendicazione, senza intermediari (fatta cioè passando sotto silenzio la bucolica latina e volgare dei secoli precedenti), della ascendenza teocritea-virgiliana della propria poesia pastorale ${ }^{34}$ 
2) il corollario della ripresa della lezione virgiliana e, con ciò, l'allusione alla propria originalità di poeta, ribadita, è noto, nel congedo del I504. Il «silenzio» che segue a Virgilio nella storia della poesia bucolica schizzata in Arcadia X 13-20 è stato registrato nei commenti fin dal Carrara $^{35}$ e in sèguito dal Saccone e dal Velli, che hanno riproposto la questione. Il brano ba poi attirato $i$ lettori quasi solo in ordine alla "genealogia della forma poetica» (Bettinzoli, p. 25) - e dunque meno, a me pare, di quello che meriti - mentre l'attenzione è corsa all'integrazione che quella «storia» riceve nella prosa XI, dove Sannazaro ricorda l'esperienza bucolica del 'napoletano', poi pontaniano, "gran pastore Panormita» (l'unico a guadagnarsi una citazione esplicita) e quelle di Petrarca, Boccaccio, forse dell'Alberti, dell'Arzocchi e del Benivieni. ${ }^{36}$ Ora non è dubbio che, facendo a modo suo la storia del genere bucolico, tacendo cioè almeno $i$ tre toscani, la Miscomini, il Boiardo e il Pulci, Sannazaro si accrediti come erede diretto di Virgilio come del resto affermerà, rivolto alla zampogna, nel congedo: "a te non picciola scusa fia lo essere in questo secolo stata prima a risvegliare le addormentate selve». Insieme, però, anche persegue, mi pare, una strategia di 'innalzamento' del genere, come appare in altri luoghi e, ancora, nel congedo A la sampogna, che di quel percorso metatestuale può considerarsi il punto d'arrivo, e come l'esplicitazione. ${ }^{37}$ La novità non risiede per tanto, come appare a prima vista, in quella speciale e difettiva genealogia bucolica quanto piuttosto nell'inserzione di un tale momento 'storiografico' entro il discorso pastorale: non dunque nella storia e rivendicazione pur abnorme del genere, quanto nella novità del registro che l'inserzione introduce nella narrazione.

Il carattere 'a chiave', allegorico e criptico della bucolica per il quale ho richiamato il Petrarca delle Sine nomine o Benvenuto commentatore di Virgilio, giustifica l'esistenza in ambito bucolico postvirgiliano di un'ulteriore piccola tradizione di testi 'esegetici' che accompagnano le egloghe senza essere dei veri e propri commenti. Cosi, limitandoci alla Toscana, il Petrarca inviando al fratello Gherardo la sua ecloga I sente il bisogno di accompagnarla con una lettera, Fam. X 4, in cui la spiega; e cosi fa con l'egloga V nelle Variae XXXVIII. Il Boccaccio, scrivendo a Martino da Signa, illustra contenuto e nomi delle sue ecloghe (Epist. V) ${ }^{38}$ e Poliziano, introducendo alla lettura di Virgilio, produce nella Manto (I48I) un elogio del poeta mantovano che è insieme una storia della poesia bucolica. In questi poeti l'esercizio bucolico si accompagna 
insomma a una 'storia' o 'esegesi' del genere. E perfino nel caso del Benivieni che, nella Firenze del Poliziano e non troppo lontano da lui, arriva a dotare $i$ suoi testi di uno straordinario apparato di commento senza il quale quei testi non si intendono, risponde tutto sommato alle modalità tradizionali della glossa. Questa tradizione, ben nota a Sannazaro (e però scarsamente richiamata nei commenti), ${ }^{39}$ è tuttavia altra cosa rispetto al brano dell'Arcadia. Risponde ancora alle modalità di una esegesi 'esterna' in forma di explicatio e glosa. Sannazaro immette, invece, la storia stessa del genere nel suo prosimetro, confrontandosi e silentio con quella tradizione. Il suo discorso pastorale assume di conseguenza una modalità decisamente 'metadiscorsiva' cui l'autore sembra affidare il compito di accompagnare la propria opera di bucolico in volgare.

\section{GLI ALBERI E IL «LIBRO»}

È possibile che il topos della 'scrittura sulla corteccia', cioè il tema del pastore che incide sull' albero 'frammenti' della propria vicenda amorosa (una parola, un nome, ecc.), sia rimasto inerte nell'interpretazione dell'Arcadia in virtu del suo essere uno dei principali stereotipi pastorali. Di per sé, il carattere di 'sterotipo' non contraddice però alla funzionalità che può avere nell'opera, e anzi vorrei mostrare che il topos si fa, nella scrittura di Sannazaro, emblema stesso del libro.

Due studiosi si sono avvicinati a un'interpretazione simile, senza però tirarne le conseguenze che qui presento: prima e soprattutto Rinaldo Rinaldi (2007), poi, più sfumatamente, Gianni Villani (2009). Rinaldi, facendo centro sulla dialettica «silenzio» / "parola» (orale o scritta) nell'Arcadia, accenna al topos della 'scrittura sul tronco' nel contesto del duplice «supporto» che l'albero e il sepolcro costituiscono per le parole del pastore d'A rcadia, ed elenca tre occorrenze del motivo nelle ecloghe I, III e XII. ${ }^{40}$ "Gli alberi - osserva - sono presenti fin dal Prologo e dalla prosa I come figura del topos edenico (sul modello boccacciano), ma si presentano anche subito come Ersatz naturale e bucolico dell' artificioso libro»; e aggiunge:

gli alberi [...], come la zampogna, rinviano al «lungo silenzio» che forma la melanconica prospettiva finale del romanzo: è un albero ad accogliere lo strumento muto nell'epilogo ed è l'allegorico «albero bellissimo di arangio [...] tronco da le radici, con le frondi e i fiori e i frutti sparsi per terra» a inaugurare oniricamente il ritorno di Sincero a Napoli. ${ }^{41}$ 
L'osservazione è acuta, ma è lasciata un po' lì e lo studioso passa poi ad analizzare le implicazioni dell'altro «supporto» della scrittura in Arcadia, il «sepolcro» (e dunque il «tumulus»), osservando come l'ultima ecloga si configuri come un

omaggio al fondamentale modello pontaniano [...], e insieme perfetta mise en abyme dello stesso romanzo sepolcrale. ${ }^{42}$

Da parte sua, Gianni Villani ha sottolineato la valenza simbolica di "alberi e piante» («sono infatti una forma intermedia e mediatrice, che punta in alto ma affonda le radici nel basso, tra cielo e terra»), condividendo la «funzione propiziatrice di scrittura esercitata dal simbolo delle piante» e osservando:

Tra la breve durata della memoria individuale e quella protratta della poesia si interpone la mediazione delle piante. $^{43}$

Credo che anche qui si possa andare oltre nellinterpretazione della funzionalità del topos. Converrà intanto osservarne la frequenza nell'Arcadia, ben più intensa delle tre occorrenze segnalate, e soprattutto studiarne la disposizione nel «libro», registrandone l'intensificazione sul finale dell'opera. Le nude occorrenze del topos sono infatti I7. Le riporto nell'ordine di apparizione e le leggo entro la crescente dialettica «scrittura» / «lettura» cui sembrano rinviare nel «libro», aggiungendo che queste sono due facce di una pratica intellettuale che - ̀̀ utile ricordare - raggiunge, dopo l'epoca medievale, il massimo di intensificazione in Occidente proprio nella riunificazione che ne attua Petrarca. $^{44}$

Si presti allora attenzione alla serie che l'Arcadia documenta $e$ all'intensificarione cui il topos è sottoposto nell'ultima ecloga:

Prol. 2 [voce narrante]

Per la qual cosa ancora, sì come io stimo, addiviene che le silvestre canzoni vergate ne li ruvidi cortecci de' faggi dilettino non meno a chi legge che li colti versi scritti ne le rase carte degli indorati libri

Ecl. I I 04 [Ergasto]

Quest'alberi di lei sempre ragionano e ne le scorze scritta la dimostrano, ch'a pianger sempre et a cantar mi spronano 
Pr. III 22 [voce narrante, all'interno di ecfrasis]

Appresso di costui era Paris, che con la falce avea cominciato a scrivere «Enone» a la corteccia di un olmo

Ecl. IV I-5 [Logisto]

Chi vuole udire i miei sospiri in rime,

$[\ldots]$

legga per queste querce e per li sassi

Pr. v I 3 [Opico]

Ove, sì come io stimo, trovaremo molti alberi nei quali io un tempo, quando il sangue mi era più caldo, con la mia falce scrissi il nome di quella che sovra tutti gli greggi amai; e credo che ora le lettere inseme con gli alberi siano cresciute

Pr. VI I [voce narrante]

Mentre Ergasto cantò la pietosa canzone, Fronimo, sovra tutti i pastori ingegnosissimo, la scrisse in una verde corteccia di faggio

Ecl. IX I 2 I-23 [Elenco]

Qualunque per amor sospira e brama

leggendo $i$ tronchi ove segnata stai,

«Beata lei - dirà - chi il ciel tant'ama!»

Ecl. XI I 5 [Ergasto]

e quant'io parlo per li tronchi scrivi

Ecl. XI 34

scrivi i miei dolori in le tue foglie $e^{45}$

Ecl. XI I00-107

Non che sia degno da notarsi in carte, ma che sol reste qui tra questi faggi così colmo d'amor, privo d'ogni arte; acciò che in questi tronchi aspri e selvaggi leggan gli altri pastor che qui verranno [...]

e poi crescendo ognor più d'anno in anno, memoria sia di lei fra selve e monti. 
Le ultime declinazioni del topos appaiono nell'egloga XII: messe in bocca principalmente a Barcinio-Cariteo (il solo poeta vivo tra gli interlocutori dell'egloga) e, in due casi, al Summonte:

Ecl. XII I-3

Qui cantò Melibeo, qui proprio assisimi, quand'ei scrisse in quel faggio: «Vidi, io misero, vidi Filli morire, e non uccisimi»

Ecl. XII IO-I I

e via più dentro il cor m'induro e inaspero pensando a quel che scrisse in un giunipero

Ecl. XII 22

Volgi in qua gli occhi e mira in su quel corilo

Ecl. XII 70-7I

scrissi i miei versi in su le poma puniche,

e ratto diventâr sorba e corbezzoli

Ecl. XII 27I-72

Summonzio, io per li tronchi scrivo e vergole, e perché la lor fama più dilatesi per longinqui paesi ancor dispergole.

$O$, più raramente, in bocca a Summonte:

Ecl. XII 26

ma cerca ben se v'è pur altro arbuscolo

Ecl. XII 244-45

Deh, se ti cal di me, Barcinio, scribili, a tal che poi, mirando in questi cortici, l'un arbor per pietà con l'altro assibili.

Si badi all'alternanza «scrizione» / «lettura», che ho detto: «canzoni vergate ne li ruvidi cortecci de' faggi», "quest'alberi [...] a cantar mi spronano», "avea cominciato a scrivere a la corteccia di un olmo», «legga per queste querce e per li sassi», "avea cominciato a scrivere», "alberi nei quali io [...] scrissi il nome di quella», "la scrisse in una verde corteccia di faggio», «leggendo $i$ tronchi ove segnata stai», "per li tronchi scrivi», 
«scrivi [...] in le tue foglie», «in questi tronchi aspri e selvaggi leggan gli altri pastor», e via dicendo con gli esempi dell'ultima egloga.

Non è dubbio che questo sia, con le sue I7 occorrenze, il topos pastorale più declinato nell'Arcadia, dove (il fatto non ha destato la giusta attenzione) l'ultima egloga da sola ne conta ben sette. Al di là, c'è l'epilogo A la sampogna, dove non solo - come ricorda Rinaldi - «èun albero ad accogliere lo strumento muto» ${ }^{46}$ ma il gesto con cui SinceroSannazaro pone fine all'attività di poeta pastorale rinvia molto precisamente a quello con cui, a metà del libro, Fronimo prima trascrive la «pietosa canzone» cantata da Ergasto su una corteccia e poi la offre alla lettura, appendendola a un albero: "Fronimo[...] la scrisse in una verde corteccia di faggio; e quella [...] appiccò a un albero» (VI I).

Cio invita, mi pare, a riflettere chiedendosi se tale presenza e poi intensificazione del motivo non significhi qualcosa di più di un «Ersatz [...] dell'artificioso libro» (Rinaldi). Credo che, soprattutto per l'accelerazione che il tema riceve in chiusa, piu che di Ersatz si tratti della cifra stessa della «scrittura» e dunque di un rinvio alla «costruzione» stessa del testo, una «figura» insomma dell'operare del poeta che, con lucida coscienza del suo "ffare», ricava da un topos pastorale per eccellenza l'emblema del suo operato. Del resto, come mi ricorda Vera Tufano (che ringrazio) un'equivalenza tra «cortex»e «librum», chiudeva già la seconda ecloga di Pontano, indicando in quei due lemmi la via per la quale il dolore di Meliseo per la perdita della moglie si sarebbe sanato:

Cortice quoque etiam lentescat vulnus et hudo, quae super ipse linens imponat glutina, libro. ${ }^{47}$

Sarà allora necessario aggiungere che proprio il Meliseo pontaniano è il testo che ispira l'ultima egloga del «libro» e leggere la terapia letteraria che propone Pontano come uno stimolo alla «scrittura» sannazariana? Una conferma a fare del topos una cifra della composizione del testo fornisce Sannazaro, quando esplicita la relazione che corre tra «crescita» della pianta e «crescita» della «scrittura». In altre parole, quando segnala che le «lettere» del messaggio inciso sul tronco sono destinate a crescere con il crescere di questo. In questo modo, il lamento che il pastore affida al tronco è destinato necessariamente, biologicamente, a farsi 'racconto'. Nei termini ridotti dell'emblema, il topos allude al processo di formazione-composizione dell'Arcadia. 
La correlazione tra 'scrittura sul tronco' e crescita delle «lettere» che propone la prosa V I3 («credo che ora le lettere insieme con gli alberi siano cresciute»), e, di nuovo, l'ecloga XI I03-I06:

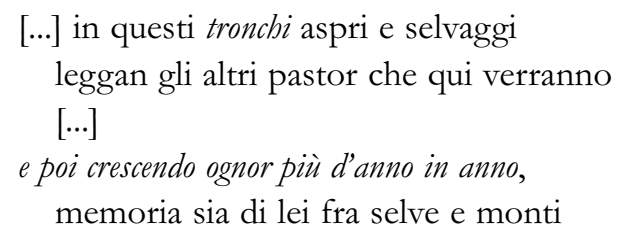

è, ovviamente, motivo già timidamente presente in Petrarca: «ove 'l gran lauro fu picciola verga / crescendo mentr'io parlo agli occhi tolle» (Rvf I88, II-I2). E si tratta, altrettanto ovviamente, di un topos antico, tra pastorale e elegiaco, che si ritrova (bastino questi due ambiti) nelle Bucoliche di Virgilio e nelle Heroides di Ovidio. ${ }^{48}$ Nell'Arcadia, esso può leggersi però, con nuova più tecnica funzione, come "figura» della «scrittura»: emblema, cioè del processo di 'crescita' che dall'esercizio di ecloghe sparse giunge alla narrazione organica. Un processo su cui, è stato detto, pesa l'esempio delle Bucoliche elegantissime della Miscomini (I482), ove il genere assume, per la prima volta (nell'ambito della letteratura in volgare) forma e dignità di «libro» e di cui probabilmente Sannazaro non ha dovuto attendere la stampa per leggerne gli autori. ${ }^{49}$

Se questa lettura è plausibile, essa implica un corollario che riguarda l'episodio famoso della morte dell" «albero bellissimo d'arangio». Sincero narra cosi la «visione» (Pr. XII 7-8):

Ultimamente un albero bellissimo di arangio, e da me molto coltivato, mi parea trovare tronco dalle radici, con le frondi e i fiori e i frutti sparsi per terra. E dimandando io chi ciò fatto avesse, da alcune ninfe che quivi piangevano mi era risposto le inique Parche con le violente scure averlo tagliato. De la qual cosa dolendomi io forte, e dicendo sovra lo amato troncone: «Ove dunque mi riposerò io? Sotto qual ombra omai canterò $\mathrm{i}$ miei versi?», mi era da l'un de' canti mostrato un nero e funebre cipresso, senza altra risposta avere alle mie parole.

In questo tanta noia e angoscia mi soprabondava che, non possendo il sonno soffrirla, fu forza che si rompesse. 
L'episodio è stato variamente interpretato e di esso due sono state le interpretazioni fornite: una politica, la sventura della dinastia aragonese (dallo Scherillo al Vecce) e l'altra amorosa, la morte della fanciulla amata da Sannazaro (dal Carrara alla Riccucci). ${ }^{50}$ Delle due, lo svelamento ad opera del Velli della fonte, costituita dall'ecloga $x$ di Petrarca, autorizza forse soprattutto la seconda. Non è tuttavia stato notato un dettaglio presente nella rappresentazione di Petrarca, che apre a una terza interpretazione, ed è la caduta del «cortex» che accompagna la morte dell'albero. Nel suo testo, Petrarca la richiama per due volte opponendo alla morte del «cortex》 la vitalità invece delle «radices» della pianta, senza che il dettaglio fosse veramente necessario come dice la sua assenza nella fonte additata dallo Scherillo (Claudiano) ${ }^{5 \mathrm{I}}$ e comune a Petrarca e Sannazaro.

Il taglio dell'arancio potrà allora essere si anche la perdita della donna amata e di conseguenza dell'occasione della poesia ${ }^{52}$ o la «scomparsa di una grande figura della dinastia aragonese» (di cui «arangio», ’̀ stato ricordato, è anagramma), ${ }^{53}$ ma è a mio avviso prima di tutto il rovesciamento catastrofico e annientatore del «cortex» inteso come supporto del lamento pastorale e, di conseguenza, della scrittura di SinceroSannazaro. La 'scrittura sul tronco' sarebbe allora qualcosa di molto vicino al progetto, che Sannazaro iscrive - si è visto - ossessivamente nel «libro» e sottolinea poi nella storia del genere posta in chiusa della prima redazione, di rinnovare forma e contenuti dell'avventura bucolica. Tale pare a me, del resto, anche il senso profondo del lamento di Sincero a XII 8: "Ove dunque mi riposerò io? Sotto qual ombra canterò $i$ miei versì.

Una domanda sorge a conclusione di questo percorso e riguarda la legittimità di un'interpretazione che promuove un topos bucolico fortunato a figura della scrittura e composizione dell'Arcadia. Risponderò brevemente, ricordando un bel contributo che vari anni fa diede Pier Cesare Bori indagando il senso e la portata di una formula presente nell'esegesi biblica di Gregorio Magno e di li passata poi alla riflessione esegetica successiva. Pur diversamente modulata, nella sua versione più sintetica la formula cosi suona: "divina eloquia cum legente crescunt», 'il testo sacro cresce con il suo lettore. ${ }^{54}$ L'immagine che abbiamo ritrovato nel Virgilio bucolico del messaggio pastorale che, iscritto sul «cortex», cresce con l'albero e dunque con chi lo legge, ${ }^{55}$ è dunque di casa nell'esegesi medievale, anche se l'ambito biblico comporta - come Bori 
ha chiarito - un'idea diversa di «crescita», strettamente implicata con l'attesa, la partecipazione e la fede del credente. Gregorio Magno nel suo commento alle visioni di Ezechiele, affermava più precisamente il principio che «l'intelligenza delle parole divine [...] cresce secondo la capacità di sentire di chi le legges: verba sacri eloquii, ut saepe dictum est, iuxta sensum legentium per intellectum crescunt, ${ }^{56}$ principio ricco di conseguenze, se tra le altre ne discende quella di un testo sacro capace di adeguarsi alla sensibilità e al livello del suo lettore (magarievangelicamente - a quello più 'semplice' piuttosto che al più 'colto') $e$ dunque della sua «attualità». L'analogia tra l'adagio di Gregorio e il topos presente nell'Arcadia non richiede troppe spiegazioni: come il testo biblico si arricchisce e riceve vita dalla comunità dei suoi lettori, cosi quello bucolico vive e cresce offrendosi al pubblico attraverso il supporto della inscriptio in cortice. Che lo abbia o meno presente, in fondo l'umanista Sannazaro non fa che adibire al terreno della poesia bucolica un'idea fiorita sul terreno dell'esegesi biblica per dar conto della ricchezza di un testo direttamente ispirato da Dio: una conversione da un ambito sacro al suo opposto, che parrà forse meno ardita se la inseriamo in quell'idea di «collaborazione» che, secondo la formulazione di Montaigne (Essais I 24), necessariamente si instaura tra il lettore e il suo testo.

Ricostruendo la genealogia della formula patristica, Bori dimostra che l'adagio di Gregorio Magno si iscrive in una «tradizione interpretativa» di lunga durata, che conosce momenti storici di pausa e irrigidimento dovuti all'esigenza della Chiesa romana, come di altre comunità ecclesiali, di recuperare un 'canone' opponendosi alla «'dilatazione' della Scrittura». La questione è ampia e complessa e di recente, se non mi sbaglio, è tornata ad affiorare nella discussione seguita a un volume di Luciano Canfora dedicato alla storia della filologia biblica. ${ }^{57}$ Senza entrare nel merito di quella discussione, e ritornando a cio che qui importa, pare interessante registrare questa convergenza di parametri dell'esegesi sacra nell'immaginario bucolico umanistico: una convergenza che - come è tornato a sottolineare Bori - può stupire solo chi non abbia presenti le ascendenze teologiche del problema ermeneutico. ${ }^{58} \mathrm{E}$, aggiungo, chi non consideri l'ampia cultura classico-antiquaria, e dunque anche biblica di Sannazaro. ${ }^{59}$

Massimo Danzi 
BIBLIOGRAFIA

Edizione critica:

Iacopo Sannazaro, Arcadia, in Sannazaro, Opere volgari, a cura di A. Mauro, Bari, Laterza, I96r.

Edizioni commentate:

IAcopo Sannazaro, L'Arcadia, a cura di E. Carrara, Torino, UTET, I948.

Iacopo Sannazaro, L'Arcadia, a cura di F. Erspamer, Milano, Mursia, i99o.

Iacopo Sannazaro, Arcadia / L'Arcadie, a cura di G. Marino. Introduzione di Y.

Bonnefoy, Paris, Les Belles Lettres, 2004.

Iacopo Sannazaro, L'Arcadia, a cura di C. Vecce, Roma, Carocci, 2013.

Atti di convegni:

Le genre pastoral en Europe du $X V^{*}$ au $X V I I^{e}$ siècle, Saint-Étienne, Publications de l'Université de Saint-Étienne, I980.

Carrai i 998, (a cura) La poesia pastorale nel Rinascimento, Padova, Antenore.

Comboni-Di Ricco 2000 (a cura), Il prosimetro nella letteratura italiana, a cura di A. Comboni e A. Di Ricco, Trento, Università di Trento.

Canfora-Caracciolo Aricò 2006 (a cura di), La Serenissima e il Regno. Nel V Centenario dell'Arcadia di Iacopo Sannazaro, Bari, Cacucci.

Sabbatino 2009 (a cura), Iacopo Sannazaro. La cultura napoletana nell'Europa del Rinascimento, Firenze, Olschki.

Saggi critici:

Beccherucci 201 2, L'alterno canto del Sannazaro. Primi studi sull'Arcadia, Lecce, Pensa MultiMedia Editrice.

Boillet I 977, Paradis perdus et retrouvés dans l'«Arcadie» de Sannazaro, in AA.VV., Ville et campagne dans la littérature italienne de la Renaissance. II. Le courtisan travesti, Études réunies par A. Rochon [...], Paris, Univ. de la Sorbonne Nouvelle.

Boudard I980, Un exemple de diffusion de la pastorale italienne en France au XVTe siècle: la première traduction française de l'Arcadia de Jacopo Sannazaro par Jean Martin, in «Bulletin de l'Association d'Études sur l'Humanisme, la Réforme et a Rénaissance», a. VI, I 2.

Brugnoli 200I, I nomi del Petrarca, in «Onomastica \& Letteratura» II-III, 2000$200 \mathrm{I}$ (Convegno internazionale di Studi «Il nome nel testo», Pisa, Università di Pisa, I7-I 8 febbraio 2000).

Caracciolo Aricò 2000, L'Arcadia del Sannazaro nell'autunno dell'Umanesimo, Roma, Bulzoni.

Caracciolo Aricò 2005, Jacopo Sannazaro Orfeo in Arcadia, in Il mito nella letteratura italiana, I Dal Medioevo al Rinascimento, a c. di G. C. Alessio, Brescia, Morcelliana.

Caracciolo Aricò 2007, Il mito di Orfeo nel mondo aragonese, in «Critica letteraria», Xxxv, 4, n. I 37.

Charis Marconi i 997, La nascita di una vulgata: l'A Arcadia del I504, Roma, Vecchiarelli. 
Carrai i 999, I precetti di Parnaso. Metrica e generi poetici nel Rinascimento italiano, Roma, Bulzoni.

Carrai, 2006, La morfologia del libro pastorale prima e dopo Sannazaro, in L'usignolo di Bembo, Roma, Carocci.

Corti 1954 , Le tre redazioni della «Pastorale» di P.J. De Jennaro con un excursus sulle tre redazioni dell" "Arcadia», in "Giornale storico della letteratura italiana», I 2 I.

Corti 1964, L'impasto linguistico dell" "Arcadia» alla luce della tradizione manoscritta, in «Studi di Filologia italiana», 22.

Corti i 969, Il codice bucolico e l" «Arcadia» di Iacopo Sannazaro [1968], in Ead., Metodi e fantasmi, Milano, Feltrinelli.

Corti 1968, Rivoluzione e reazione stilistica nel Sannazaro, [1968], in Ead. Metodi e fantasmi, cit..

De Robertis I966, L'Arcadia del Sannazaro, in Storia della letteratura italiana, Milano, Garzanti, vol. IV.

De Robertis i98 I, L'ecloga volgare come segno di contraddizione, in «Metrica», II.

Fenzi 2008, L'impossible Arcadia di Iacopo Sannazaro, in «Per leggere», Is (poi in Sabbatino 2009).

Fenzi 2009, Arcadia X-XII, in Travestimenti: mondi immaginari e scrittura nell'Europa delle corti, a cura di R. Girardi, Bari, Edizione di Pagina.

Folena 1952, La crisi linguistica del Quattrocento e l'Arcadia di Iacopo Sannazaro, Firenze, Olschki.

Gaietti 1977, Edipo in Arcadia Miti e simboli nell" «Arcadia» di Sannazaro, Napoli, Guida.

Gargano 2006, L'Arcadia di Jacopo Sannazaro in Spagna, in Sabbatino 2009.

E. Haywood 2006, «E che parlo io? E chi mi ascolta?». Poeti e pubblico nell'Arcadia di Iacopo Sannazaro, in Canfora-Caracciolo Aricò 2006.

De las Nieves Muñiz Muñiz 2007, Dopo Valla: fonti greche latine e 'locus amoenus'. Un caso di intertestualità tra Sannazaro e Garcilaso, in Valla e Napoli. Il dibattito filologico in età umanistica. Atti del Convegno internazionale, Ravello, Villa Rufolo, 22-23 settembre 2005 , a cura di M. Santoro, Pisa-Roma, Istituti editoriali e poligrafici internazionali.

De las Nieves Muñiz Muñiz 20 I I, Sulla tradizione della «descriptio puellae» e sull'Amaranta di Sannazaro, in «Rinascimento meridionale», II.

Lavocat 2005, La syrinx au bûcher. Pan et les Satyres à la Renaissance et à l'âge baroque, Genève, Droz.

Marino 2007, Itinéraires de Sannazaro en Arcadie. L'héritage de Virgile (de Gallus et Orphée à Aristée), in «Lettere italiane», 2.

Parenti i979, «Antonio Caracciolo desamato». Aspetti della poesia volgare aragonese nel ms. Riccardiano 2752, in «Studi di filologia italiana», 32.

Parenti 1987, La poesia pastorale come poesia artificiosa. Origine e fortune del Summationsschema, in «Colloquium Helveticum», 6.

Prandi 2004, L'ecfrasi pastorale in L'ecfrasi. Modelli ed esempi fra Medioevo e Rinascimento, a cura di G. Venturi e M. Farnetti, Roma, Bulzoni, vol. II. 
Quint 1983, Sannazaro: from Orpheus to Proteus, in Origin and Originality in Renaissance Litterature, New Haven and London, Yale U.P.

Riccucci 200I, Il Neghittoso $e$ il Fier connubbio. Storia e filologia nell'Arcadia di Jacopo Sannazaro, Napoli, Liguori

Rinaldi 2007, Dal silenzio al ricordo. Conquista della scrittura nell'Arcadia, in «Critica letteraria», xxxv, 2, n. I 35 .

Saccone i 969, L'Arcadia di Iacopo Sannazaro: storia e delineamento di una struttura, in «MNL», 84, I.

Santagata 1979, L'alternativa «arcadica» del Sannazaro, in La lirica aragonese. Studi sulla poesia napoletana del secondo Quattrocento, Padova, Antenore.

Scattolin 2006, Dante in Arcadia. Presenze di Dante volgare nell'Arcadia di Jacopo Sannazaro, in Canfora-Caracciolo Aricò 2006.

Tateo I967, La crisi culturale di Jacopo Sannazaro, in Tradizione e realtà nell'Umanesimo italiano, Bari, Dedalo Libri, 1974 (I ed. 1967).

Tateo ${ }_{19} 67$ Il mondo arcadico e i temi della cultura umanistica, Ibid..

Tateo 2004, Filologia e immaginazione nell'onomastica sannazariana, «Il Nome nel testo» VI, I (Atti del IX Convegno internazionale di Onomastica e Letteratura).

Vecce 1999, L’Arcadie de Sannazar, selon Jean Martin, in Jean Martin un traducteur au temps de François Ier et de Henri II, Paris, Presse de l'École Normale supérieure («Cahiers V.L. Saulnier», I6).

Vecce 2000 , Il prosimetro nella Napoli el Rinascimento, in Il prosimetro nella letteratura italiana, a cura di A. Comboni e A. Di Ricco, Trento, Università di Trento.

Velli 1983, Sannazaro e le «Partheniae myricae»: forma significato dell" «Arcadia», in Tra lettura e creazione. Sannazaro, Alfieri, Foscolo, Padova, Antenore.

Villani 1989, Per l'edizione dell' «Arcadia» del Sannazaro, Roma, Salerno Editrice.

Villani 992, L'Arcadia di Iacopo Sannazaro, in Letteratura italiana. Le Opere, vol. I, Torino, Einaudi.

Villani 2009, Processi di composizione e 'decomposizione' nell'Arcadia di Sanazaro, in «Nuova rivista di letteratura italiana» XII, I-2.

I. Si veda la bibliografia in appendice, selettiva ma, mi auguro, non arbitraria.

2. Recentemente Vecce 2013 tiene invece a base del suo commento una ristampa del I 507 , considerata portatrice di varianti probabilmente autoriali: per una valutazione di questo e altri aspetti del commento, rinvio alla scheda data in «Bibliothèque d'Humanisme et Renaissance» LXxv, 2, 2013 , pp. 384-88.

3. Nei saggi di Tateo 2004, della Muñiz e ancora nel commento di Vecce. Da ultimo, questo aspetto è centrale anche nelle pagine che Santagata ha dedicato al Boiardo bucolico, nel saggio uscito dal Mulino nel 2016. E si ricordi, per i nomi pastorali di Petrarca, il contributo di Brugnoli $200 \mathrm{I}$.

4. Senza entrare nel dettaglio di questi studi, ricordo che, fino all'apparizione dell'edizione del Mauro, crescono in genere sul testo proposto dallo Scherillo nel I 888, ricco anzi esorbitante nel commento ma contaminatorio sul piano filologico: una contaminazione che oggi sappiamo aggravata dalla distanza che inter- 
corre fra le testimonianze di redazioni distinte (il codice vaticano e la princeps del I 504), una contaminazione che non era comunque sfuggita alle attenzioni del Folena e della Corti.

5. Ma vanno ricordati gli importanti contributi di Giovanni Parenti sulla poesia aragonese (con le monografie sul Caracciolo del i 979, sul Pontano del i 985 e sul Cariteo del i 993) e, in particolare, con gli studi ricordati d'ambito più sannazariano richiamati in bibliografia: uno dei quali, il Caracciolo, per essere uscito in rivista nello stesso anno della monografia di Santagata, è troppo poco ricordato.

6. Con più decisa identificazione di Santagata del De Petruciis col «neghittoso» di Ecl. x I 23 .

7. Fenzi 2008, pp. 162-63 e Fenzi 2009, p. 49.

8. Questa la sintesi che lo studioso propone dell'Arcadia: «da una parte una crisi nei rapporti sociali, dall'altra la denuncia di una crisi che investe l'attività poetica. I due [temi] insieme si collegano strettamente come aspetti di una più generale crisi dei valori, che ha il proprio referente storico nella situazione politica venutasi a creare nel Regno con la congiura dei baroni» (Santagata I979, p. 342).

9. Fenzi 2008, p. I58. E qualche pagina dopo: «Non che il 'genere' [bucolico] possa essere privato de suoi diritti, esibiti in qualche caso in maniera evidente [...] ma affogare in esso l'elemento storico reale che determina tanta parte dell'ispirazione e degli èsiti dell'Arcadia sarebbe, a parer mio, prova di grave incomprensione» (Fenzi 2008, p. I66). Siamo, dunque, agli antipodi di quanto a proposito delle identificazioni dello Scherillo di certi personaggi (Ergasto-Sanazaro e Massilia-Masella) scriveva, per es. Tateo I974, p. 60 n. 25 : «A noi non interessa riprendere il tema delle 'allusioni' storiche dell'Arcadia [...] non perché questo tema appare senz'altro secondario, soprattutto per l'interpretazione di un libro come l'Arcadia, ma perché riteniamo che il poeta abbia inteso appunto sfumare i contorni reali di quell'amata figura [scil. Massilia-Masella, madre del poeta] per accentuarne i caratteri simbolici». E la tensione verso l'interpretazione «metapoetica» anima decisamente, e molto giustamente (a mio avviso), anche il bel commento di Cristina Montagnani alle Pastorale di Boiardo (201 5).

io. Francesco Petrarca, Sine nomine. Praefatio (ed. a cura di U. Dotti, Bari Laterza, I 974, p. 3). La citazione da Benvenuto è da F. Ghisalberti, Le chiose virgiliane di Benvenuto da Imola, in Studi virgiliani pubblicati in occasione delle celebrazioni bimillenarie della Reale Accademia Virgiliana, Mantova, Reale Accademia Virgiliana, I930, p. I 77 n. I.

I I. Come è evidente, una tantum, nel canto di Caracciolo, Ecl. x i 57-59: «Gran cose in picciol velo oggi restringo: / io ne l'aria dipingo, e tal si stende, / che forse non intende il mio dir fosco» (e, in parallelo, nella prosa che lo precede: $p r$. $\mathrm{x} 7$ ).

I 2. Fenzi 2008 , p. 172 che parla di «quella turba di funzionari e affaristi catalani, di cui la corte in tanto bisogno di uomini pensava di potersi fidare e che ora, qui, a Napoli, vive di preda». Fondamentali per questa interpretazione sono gli studi sul ruolo e la presenza catalana nel Reame dello storico Mario del Treppo, ivi citati. 
I3. Fenzi 2008, p. 172 .

I4. Santagata 1979, cap. III e Fenzi 2008, p. 173.

I 5 . Esilio al quale sono pronti però anche altri pastori, come è il caso di Caracciolo: cfr. Ecl. x i 89-93: «E se se non fusse che 'l suo gregge affrenalo / e tienlo a forza ne l'ingrata patrïa, / che a morte desiar spesso rimenalo / verrebbe a noi, lassando l'idolatrïa / e gli ombrati costumi al guasto».

I6. Ł̀ il caso di Vecce, che a ogni partizione del testo fa seguire una scheda con considerazioni interpretative più ampie.

I7. Acquisita la centralità della princeps summontina (che tuttavia Vecce ora sacrifica in favore di una ristampa, giudicata portatrice di varianti d'autore), la studiosa evidenzia simmetrie e antitesi nella costruzione del romanzo, attraverso una lettura 'interna' del libro. Meno necessaria mi pare la proposta «filologica» di una diversa segmentazione paragrafematica del testo, che dia ragione della contiguità postulata tra le prose VII e viII: cfr. Beccherucci 20I2, pp. I 28 e ss.

I 8. M. Corti, Rivoluzione e reazione stilistica nel Sannazaro [1968], che leggo in Nuovi metodi e fantasmi, Milano, Feltrinelli, 200 I, p. 307.

19. Basti qui rinviare alla linea rappresentata dagli studi di Giovanni Pozzi e in particolare al suo Temi, tópi, stereotipi in Letteratura italiana, vol. III. Le forme del testo, I. Teoria e poesia, Torino, Einaudi, I984, pp. 391-436.

20. Y. Bonnefoy, L'Arcadie et la réflexion sur la poésie, in Iacopo Sannazaro, Arcadia / L'Arcadie. Introduction, traduction, notes et tables par G. Marino. Avec une préface de Y. Bonnefoy, Paris, Les Belles Lettres, 2004, p. IX.

21. Villani 2009, p. 74.

22. Del tipo: «a te [sampogna mia] non picciola scusa fia lo essere in questo secolo stata prima a risvegliare le addormentate selve e a mostrare a' pastori di cantare le dimenticate canzoni» (SANnAZAro, $A$ la sampogna, i 5). Su altre basi, il tema è stato parzialmente toccato dal Saccone, fin dal 1969.

23. Caracciolo Aricò 2000, pp. 59-62 e Caracciolo Aricò 2005, pp. 40-4I, che però insiste soprattutto sul nesso tra il tema di Orfeo e il compianto per la madre (di Ergasto), proponendo a modelli alcuni epicedî di Stazio e una epistola consolatoria dell'Altilio per la morte della madre di Sannazaro. Bibliografia sul tema, per altro ridotta sul fronte della poesia volgare, in Caracciolo Aricò 2007, p. 629 n. Io, saggio che indaga la fortuna del tema presso altri poeti aragonesi. Più in generale, per l'ambito italiano (se pur Sannazaro non compare) la tesi dottorale di Laura Rietveld, Il trionfo di Orfeo: la fortuna di Orfeo da Dante a Monteverdi (University of Amsterdam, 2007, disponibile on line).

24. A. Tissoni Benvenuti, L'Orfeo del Poliziano. Con il testo critico dell'originale e delle successive forme teatrali, Padova, Antenore, I986, pp. 73-74. Per le fonti e principali interpretazioni del tema, non solo in relazione a Poliziano, si vedano ancora le pp. 74-80. 
25. Composto nel I486, fu edito sol nel i49I. Su questo tema dei Nutricia, a confronto con la Favola d' Orfeo e le sue fonti, si veda almeno G. Boccuto, Il mito di Orfeo nei «Nutricia» di Poliziano, in Il mito nel Rinascimento, a cura di L. Rotondi Secchi Tarugi, Milano, Nuovi Orizzonti, 1993, pp. 2 17-40.

26. Erspamer: Teocrito, Virgilio, Georg., Ovidio Metam., Claudiano, Boccaccio, Poliziano ecc.; Vecce: Virg., Ov., e soprattutto Boccaccio. La fonte di Eliano, aggiunta ultimamente da Marino 2007, non interessa invece il tratto del catalogo arboreo.

27. Ecl. Iv 45-46: «Deh, se ciò fusse [che giungesse finalmente un lieto giorno, da «cantar più dolci rime»: 36], or qual mai piaggia / o valle udrebbe tante sì süavi rime? / Certo io farei saltar i boschi e i sassi / sì come un tempo Orfeo col dolce pianto».

\section{Caracciolo Aricò 2009.}

29. Ecl. xi 64-75: «Felice Orfeo che, innanzi l'ore extreme, / per ricoprar colei che pianse tanto / securo andò dove più andar si teme! / [....] / Or perché al suon di curvo legno / temprar non lice a me sì meste note, / ch'impetri grazia del mio caro pegno? / E se le rime mie non son sì note / come quelle d' Orfeo, pur la pietade / dovrebbe farle in ciel dolci e devote». Leggendo l'Arcadia come un organismo attraversato da analogie e simmetrie, Marino 2007 distingue ulteriormente le fruizioni del mito di Ergasto e di Sincero.

30. Come ha visto, sulla base di altri elementi, già Tateo per il quale la morte della fanciulla (cui allude l'episodio dell'«albero bellissimo di arangio» della pr. xII) è anche la morte della poesia: ma per ciò vedi il terzo punto di questo mio intervento.

3 г. Georg. IV 5 го-г 3: «septem illum [Orfeo] [...] menses / [...] / flesse sibi et gelidis haec evolvisse sub astris / mulcentem tigris et agentem carmine quercus, / qualis populea maerens philomela sub umbra / amissos queritur fetus, quos durus arator / observans nido implumis detraxit». La fonte è nota ma la particolare allusività di Sannazaro consiste, come finora non è stato osservato, nel ricondurre il topos all'episodio di Orfeo.

32. Ovidiane col tema di Alfeo e Aretusa, virgiliane e dantesche (su queste, da ultimo, si veda Scattolin 2006).

33. A la sampogna, I: «lungo silenzio e forse eterna quiete». Si veda per i Pastoralia l'edizione di S. Carrai (Padova, Antenore, I 996) e per le Pastorale la recentissima di C. Montagnani in Pastorale Carte e Trionfi, a cura di C. Montagnani e A. Tissoni Benvenuti, Novara, Interlinea, 20 i 5.

34. Su questo silenzio, si è interrogato Saccone, spiegandolo sulla linea del Carrara con la volontà di «restaurare» della lezione virgiliana.

35. Così il Carrara, fin dal i 926 (ma cito dalla rist. del 1970): «da buon umanista il Sannazaro non riconosceva valore ai tentativi bucolici in volgare del suo tempo né forse del tempo precedente (del Petrarca e del Boccaccio) in latino». 
36. Credo sia Saccone 1969 il primo a spiegare, pp. 47-49, il «silenzio» fatto calare sulla storia della poesia bucolica dopo Virgilio con la coscienza del Sannazaro d'essere il primo a «restaurare» l'egloga virgiliana; Velli, riempie in certo senso questo silenzio prima identificando il pastore Tirsi con l'Alberti, poi collegando il brano «storiografico» all'elenco di pastori di Arcadia xi 6o-63, ove Opico (sul quale Sannazaro proietterebbe «la propria coscienza costruttiva») dice di aver vinto «Crisaldo, figliuolo di Tirreno» [l'Arzocchi], nonché «il famoso Silvio» [Petrarca], di essersi lasciato indietro «Idalogo et Ameto» [Boccaccio] e di essere, infine, stato «superato [solo] da Tirsi, per il quale Velli propone l'Alberti (Velli I 983 , pp. 4-8, cit. a p. 6). Da ultimo, un cenno è anche in Haywood, che legge il brano all'interno della «rispondenza tra poeti e pubblico», inseguita nel suo saggio (Haywood 2006, p. 39) e in Fenzi 2009, p. 36 e poi pp. 55 e 60 per il complementare elenco di «pastori»-poeti di Arcadia xi 6o-63.

37. Dove è noto che Sincero riconosce, in barba a ogni parametro e codificazione storico-letteraria, di essere venuto in Arcadia «non come rustico pastore ma come coltissimo giovene» (A la sampogna, i6).

38. Ed è ragionevole che la condanna che Boccaccio fa della bucolica postvirgiliana (dichiarando ignobiles quei tentativi e salvando solo il maestro suo Petrarca) giustifichi in parte il silenzio del Sannazaro, come ipotizza Saccone i969, p. 49.

39. Erspamer, nota a $A r c$. x I 5 , è il solo a ricordare come «La mitica storia del genere bucolico è narrata anche da Poliziano nelle Sylvae (I: Manto)».

40. Unite, a mio avviso, all'elemento meno pertinente delle «due grandi tavole di faggio, scritte in rusticane lettere» della prosa $\mathrm{x}$.

4I. Rinaldi 2007, p. 282.

42. Rinaldi 2007 , p. 284.

43. Villani 2009, pp. 56 e 57.

44. Lo sottolinea bene, da ultimo, L. Marcozzi nella voce Biblioteca, in Lessico critico petrarchesco, a cura di L. Marcozzi e R. Brovia, Roma, Carocci, 2016, p. 78.

45. Che è l'ovidiano «ipse [Phebus] suos gemitus foliis inscribit» (Met. x 2 I 5 ), più che il «littera] inscripta est foliis, haec nominis, illa quaerela» (Met. xIII 384), ricordato dai commenti.

46. Rinaldi 2007 , p. $28 \mathrm{I}$.

47. «Anche così può rimarginarsi la sua ferita: con la corteccia e l'umido libro, su cui egli spalmi poi una mano di colla»: traduzione di Liliana Monti Sabia, in Poeti latini del Quattrocento, a cura di F. Arnaldi, L. Gualdo Rosa e L. Monti Sabia, Milano-Napoli, Ricciardi, I964, p. 387.

48. Le traduzioni sono rispettivamente di Enzio Cetrangolo (Firenze, Sansoni, i 966 ) e di Gabriella Leto (Torino, Einaudi, I 970). Virg., Ecl. v I 3-I 4: «Immo haec, in viridi nuper cortice fagi / Carmina descripsi et modulans alterna notavi, 
/ experiar» ('Cantare potrei questi versi, che dianzi sul tronco / di un faggio ho inciso insieme alla musica"); Ecl x 52-54: «[lbo et, Chalcidico quae sunt mihi condita versu / carmina, pastoris Siculi modulabor avena.] / Certum est in silvis inter spelea ferarum / malle pati tenerisque meos incidere amores / arboribus crescent illae, crescetis, amores» ('Meglio soffrire tra boschi / in antri di fiere, incider parole d'amore / su tenere piante; e cresca con gli alberi amore'). E Ovidio, Her. v 2 I-23 (Enone, sposa di Paride, scrive al marito infedele): «Incisae servant a te mea nomina fagi / et legor Oenone falce notata tua, / et quantum trunci, tantum mea nomina crescunt» ('Il mio nome che inciso hai tu, serbano $\mathrm{i}$ faggi / Dalla tua falce scritto sin può leggere «Enone». / E crescono i miei nomi come crescono i tronchi').

49. L'ipotesi, solida, è di Serena Fornasiero: cfr. la sua ed. critica commentata delle egloghe dell'Arzocchi, Bologna, Commissione per i testi di Lingua, I995, pp. XXI-XXII. Sull'importanza della stampa Miscomini si veda anche Carrai i 999, p. 137 (per le Pastorale di Boiardo) e ora Merlini, nella introduzione alla ristampa anastatica, cit.

50. Ricordano le due interpretazioni quasi tutti i saggi e commenti: e di recente, con bibliografia annessa, Caracciolo Aricò 2000, pp. 59 e 63; Pantani 2003, p. I 58 n. 46; Fenzi 2009, pp. 6o-6r; Villani 2009, p. 58 n.30; Vecce 201 3, p. 291.

5 I. PETR. Ecl x 40I-402 «pars corticis illa caduci / oppetiit, pars radices vivacior egit» e 389-9r: «Que me terra capit? Potes ad tua damna reverti, / infelix, sparsasque solo conquirere frondes, / et laceros ramos, et iam sine cortice truncum / amplecti, lacrimisque arentia membra rigare» ('Quale terra mi accoglierà? Forse potresti, infelice, tornare nel luogo delle tue sventure, cercare le fronde sparse al suolo e i rami spezzati, abbracciare il tronco privo di corteccia'. Ed. a cura di L. Canali. Collaborazione e note di M. Pellegrini, Lecce, Manni, 2005). Claudiano, De rapt. Pros., III 74-79.

52. Secondo l'interpretazione che dell'Arcadia ha dato Tateo I967, p. I 9: «il poeta raccontando la storia del suo amore, racconta in realtà la storia della sua poesia».

\section{Da ultimo, Vecce ad locum.}

54. C. Bori, Attualità di un detto antico? "La sacra scrittura cresce con chi la legge», in «Intersezioni» a. VI, n. I, aprile I986, pp. I 5-49.

55. Si veda la nota 48 , da cui riporto per comodità i soli stralci dal Virgilio bucolico: «pati tenerisque meos incidere amores / arboribus crescent illae, crescetis, amores» e dall'Ovidio delle Heroides: «et quantum trunci, tantum mea nomina crescunt».

56. Bori, Attualità di un detto antico?, cit., p. 20.

57. L. Canfora, Filologia e libertà. La più eversiva delle discipline, l'indipendenza di pensiero $e$ il diritto alla verità, Milano, Mondadori, 2008; sullo studio di Canfora sono intervenuti lo storico Franco Cardini con un testo ospitato nel suo blob http://www.francocardini.net (oggi non più leggibile) e, ai nostri fini più perti- 


\section{Massimo Danzi}

nente, Francesco Bausi, Filologia e verità, in «Ecdotica» Io (2013), pp. i 80-2 I 5 , proponendo l'insufficienza di una filologia strettamente intesa per un testo 'ispirato' indirettamente da Dio.

58. Bori, Attualità di un detto antico?, cit., p. 4I.

59. C. Vecce, Gli zibaldoni di Iacopo Sannazaro, Messina, Sicania, I998. E per l'autore del De partu Virginis si veda almeno (con l'edizione procurata da Ch. Fantazzi e A. Perosa, Firenze, Olschki, I988) M. Deramaix, Manifesta signa. Théologie et poétique, hypotypose et ekphrasis dans le De partu Virginis de Sannazar, in Canfora-Caracciolo Aricò 2006, pp. 173-202. 